\title{
Higher rank antisymmetric tensor fields in Klebanov-Strassler geometry
}

\author{
Ashmita Da:* and Soumitra SenGupta \\ Department of Theoretical Physics, \\ Indian Association for the Cultivation of Science, \\ $2 A$ \& $2 B$ Raja S.C. Mullick Road, \\ Kolkata - 700 032, India.
}

\begin{abstract}
In string theory, higher rank antisymmetric tensor fields appear as massless excitations of closed strings. Till date there is no experimental support in favour of their existence. In a stringy framework, starting from a warped throat-like Klebanov-Strassler geometry, we show that all the massless higher rank antisymmetric tensor fields are heavily suppressed due to the background fluxes leading to their invisibility in our Universe.
\end{abstract}

\section{INTRODUCTION}

A complete theoretical framework for quantisation of gravity and unification of four fundamental interactions is a long standing problem for decades. Till date many theories have been proposed in this regard. Among different proposals, string theory is elegantly designed to search for a complete theoretical understanding of these basic aspects. It is well known that in the low energy effective theory, superstring theories contains many massless higher rank antisymmetric tensor fields as different excitations of string. Among such antisymmetric tensor fields, the rank two antisymmetric KR field has some special features in the context of the spacetime geometry. It has been argued that the corresponding rank three antisymmetric field strength tensor $H_{\mu \nu \lambda}(x)$, can be interpreted as the antisymmetric connection (known as torsion) of the underlying spacetime [1].

A long awaited answer for the question that why torsion has no visible effects on our Universe (known as visible 3brane) while curvature has dominating impacts, has been addressed by the author of [2] in the background of the Randall-Sundrum (RS) [3] warped spacetime geometry. Subsequently it was shown by the same authors that all the other higher rank antisymmetric tensor gauge fields in general are also suppressed on our brane due to the warped character of RS geometry [4].

In this context it is worthwhile to re-examine this feature in the backdrop of a warped geometry originating from a stringy compactification.

Here we consider a special kind of warped compactification of 10-dimensional type IIB supergravity in presence of background fluxes, which is popularly known as Klebanov-Strassler (KS) geometry. Analysing the behaviour of various higher rank antisymmetric tensor gauge fields in the background of Klebanov-Strassler geometry we try to address the issue of non-visibility of these gauge fields in our observable Universe.

\footnotetext{
* tpad@iacs.res.in

$\dagger$ tpssg@iacs.res.in
}

\section{KLEBANOV-STRASSLER GEOMETRY: A BRIEF DESCRIPTION}

In string theory Klebanov-Strassler warped throat-like spacetime geometry [5] plays a crucial role in addressing many fundamental questions. Many authors in recent past have shown that the presence of warped throat region with D3 and anti-D3 branes can offer an explanation to different inflation and slow-roll models [6 6 -16]. Moreover this warped throat-like deformation of spacetime produces a geometrical resolution to the gauge hierarchy problem where all the low energy phenomena are confined within a highly red shifted region. This region is identified as infrared brane (IR brane), located near the tip of the throat [17.

KS geometry is an example of a warped compactification of 10-dimensional type IIB supergravity in presence of background fluxes [5]. The resulting spacetime geometry is the warped product of the 4-dimensional Minkowski spacetime with 6-dimensional Calabi-Yau (CY) orientifold. The origin of $\mathrm{KS}$ geometry is described in [57, 14, 15, 17] by considering a compactification of string theory on $A d S_{5} \times X_{5}$ where $X_{5}$ is the Einstein manifold in five dimensions. In case of $\mathrm{KS}$ geometry the above compactification reduces the background spacetime to, $A d S_{5} \times T^{1,1}$ where $T^{1,1} \sim S^{2} \times S^{3}:$ a 5 -D Einstein spacetime. Considering the radial direction present in $A d S_{5}$, the resulting six dimensional internal space has an end in the form of a tip which is joined to an $S^{3}$ of finite size and a warping can be produced by the background fluxes, making the spacetime geometry as a deformed conifold. The background fluxes are [7]:

$$
\frac{1}{2(\pi)^{2} \alpha^{\prime}} \int_{A} F=M, \quad \frac{1}{2(\pi)^{2} \alpha^{\prime}} \int_{B} H=-K
$$

Here A cycle is in the form of $S^{3}$ at the tip of the conifold and $\mathrm{B}$ cycle is the Poincare dual three-cycle. $M, K>>$ 1 are integers. In this setup the spacetime coordinates are, 4-D Minkowski coordinates and radial coordinate $r$ symbolises the distance from the tip of the conifold. In addition five more angles define the orientifold $T^{1,1}$. The metric ansatz for this KS geometry can be written as [7],

$$
d s^{2}=h^{-1 / 2} \eta_{\mu \nu} d x^{\mu} d x^{\nu}+h^{1 / 2}\left(d r^{2}+r^{2} d s_{T^{1,1}}^{2}\right)
$$


where,

$$
h(r)=\frac{27 \pi}{4 r^{4}} \alpha^{\prime 2} g_{s} M\left(K+g_{s} M\left(\frac{3}{8 \pi}+\frac{3}{2 \pi} \ln \left(\frac{\mathrm{r}}{\mathrm{r}_{\max }}\right)\right)\right)
$$

Defining,

$$
R^{4}=\frac{27}{4} \pi g_{s} N \alpha^{\prime 2}, \quad \quad N \equiv M K
$$

and neglecting the second and the logarithmic terms on the right side of eqn(3) [7], the warp factor reduces to

$$
h(r)=\frac{R^{4}}{r^{4}}
$$

We restrict our analysis in the region $r_{0}<r<r_{\max }$ of the $\mathrm{AdS}_{5}$. It is shown in [5 7, 14, 15, 17], that the slicing of the $A d S_{5}$ region within $r_{0}$ and $r_{\max }$ is a consequence of the compactification of the spacetime where away from the region $r_{0}<r<r_{\max }$, one obtains a geometry which is significantly dissimilar from the $A d S_{5} \times T^{1,1}$. Therefore the presence of the warped throat region can distinctly be identified from the embedding spacetime geometry. In the ultraviolet (UV) limit i.e $r \geqslant r_{\max }$, the throat geometry becomes conical and smoothly connects with the CY orientifold. At this connecting region the geometry deviates from $A d S_{5} \times T^{1,1}$ so that $r_{\max }$ can be projected as the ultraviolet cut-off in the Anti de-Sitter spacetime 5 7, 14, 15, 17]. Simultaneously the throat terminates smoothly in the infrared (IR) region i.e the region of small $r$ and in particular connects to an $S^{3}$ of finite size at $r=r_{0}$. The above description of KS geometry implies that if we exclude $T^{1,1}$ from the background spacetime geometry, the remaining $A d S_{5}$ is similar to RS warped geometry defined in five dimensions [7]. Therefore like five dimensional RS scenario, we identify the two end points of this trimmed $A d S_{5}$ spacetime as the location of UV brane/Planck brane (at $r=r_{\max }$ ) and IR brane/standard model (SM) brane (i.e at $r=r_{0}$ ). We focus into the region $A d S_{5}$, which contains the 4-D Minkowski and a radial coordinate, and choose to ignore the angular coordinates defined on $T^{1,1}$ orientifold from our discussion. In the background of the KS throat geometry, the authors of [17], has evaluated the redshift at the tip of the conifold, located at $r_{0}$. The derived minimal redshift is dependent on the background fluxes $M$, $K$ and can be written as,

$$
\frac{r_{0}}{R}=e^{-2 \pi K / 3 g_{s} M}
$$

\section{ANALYSIS OF MASSLESS SECOND RANK KR TENSOR FIELD IN THE BACKGROUND OF THE KS THROAT GEOMETRY}

The $A d S_{5}$ metric in KS throat geometry can be written as,

$$
d s^{2}=h^{-1 / 2}(r) \eta_{\mu \nu} d x^{\mu} d x^{\nu}+h^{1 / 2}(r) d r^{2}
$$

The five dimensional action for the Kalb-Ramond field is [2],

$$
S_{5}=\int d^{5} x H_{M N L} H^{M N L}
$$

where each Latin and Greek index runs from 0 to 4 and 0 to 3 respectively.

As discussed in [2], we consider the source of torsion to be the rank-2 anti-symmetric Kalb-Ramond field $B_{M N}$. Torsion can be identified with the rank-3 antisymmetric field strength tensor $H_{M N L}$ which is related to the $\mathrm{KR}$ field as,

$$
H_{M N L}=\partial_{[M} B_{N L]}
$$

Following the gauge symmetry, $B_{M N} \rightarrow B_{M N}+\partial_{[M} \Lambda_{N]}$, we use the gauge condition $B_{4 \mu}=0$. The Kaluza-Klein (KK) decomposition for the remaining components of KR field is now given as,

$$
B_{\mu \nu}(x, r)=\sum_{n=0}^{\infty} B_{\mu \nu}^{n}(x) \frac{\chi^{n}(r)}{\sqrt{r_{c}}}
$$

where $r_{c}$ is the distance between the UV and IR brane and $\chi^{n}(r)$ is the wavefunction for the KR field along the radial direction. Substituting this in the 5-dimensional action and integrating over the radial direction, the four dimensional effective action turns out to be:

$$
\begin{aligned}
& S_{H}^{(4)}=\sum_{n=0}^{\infty} \int d^{4} x {\left[\eta^{\mu \alpha} \eta^{\nu \beta} \eta^{\lambda \gamma} H_{\mu \nu \lambda}^{n} H_{\alpha \beta \gamma}^{n}\right.} \\
&\left.+3 m_{n}^{2} \eta^{\mu \alpha} \eta^{\nu \beta} B_{\mu \nu}^{n} B_{\alpha \beta}^{n}\right]
\end{aligned}
$$

provided the internal components of the KR field $\chi^{n}(r)$ satisfy,

$$
-\partial_{r}^{2} \chi^{n}(r)=m_{n}^{2} \chi^{n}(r) h(r)
$$

along with the orthonormality condition:

$$
\frac{1}{r_{c}} \int_{r_{0}}^{r_{\max }} h^{3 / 4(r)} \chi^{m}(r) \chi^{n}(r) d r=\delta^{m n}
$$

Here $m_{n}$ represents the KK mass modes of KR field.

Let us now consider the massless mode of the KR field which is related to the spacetime torsion. Eq.(12) yields the solutions for the internal component $\chi^{0}(r)$ of $\mathrm{KR}$ massless mode as,

$$
\chi^{0}=\sqrt{2 r_{c}} \frac{r_{0}}{R^{3 / 2}}\left(1-\frac{r_{0}^{2}}{r_{\max }^{2}}\right)^{-1 / 2}
$$

Using eqs.(44) and eq.(6) in eq(14), we obtain the wavefunction for the massless mode of KR field in terms of the background fluxes on the IR brane located at $r_{0}$ as,

$$
\chi^{0}=\sqrt{2} e^{-\pi K / g_{s} M}\left[\frac{r_{\max }}{r_{0}}\left(1+\frac{r_{\max }}{r_{0}}\right)^{-1 / 2}\right]
$$


In [2], it was shown that in the background of the RS warped geometry, the zeroth mode of the KR field (which is related to the spacetime torsion in 4-D spacetime) is heavily suppressed on the low energy brane. This results into a heavy suppression of it's couplings to the brane fields (or SM fields) in comparison to the corresponding couplings of the massless 4-D graviton.

Here in string compactification, eq. (15) is again exhibiting an exponential suppression of the massless KR field on the IR region and this suppression depends on the background flux parameters.

We now determine the coupling of the torsion field with the standard model (SM) spin-1/2 fermion fields, which are confined to the low energy brane located at $r=r_{0}$ in the IR region. The interaction Lagrangian of the massless KR field and the SM fermions is,

$$
\mathscr{L}_{\psi \bar{\psi} H^{0}}=\frac{1}{M^{3 / 2}} \bar{\psi}\left[i \gamma^{\mu} \sigma^{\nu \lambda} H_{\mu \nu \lambda}^{0}\left(x^{\mu}\right) \frac{\chi^{0}(r)}{\sqrt{r_{c}}}\right] \psi
$$

Integrating over the extra dimensional part, the effective 4-D Lagrangian becomes,

$$
\mathscr{L}_{\psi \bar{\psi} H^{0}}=i \bar{\psi} \gamma^{\mu} \sigma^{\nu \lambda}\left[\frac{e^{-4 \pi K / 3 g_{s} M}}{M_{P l}}\left(\frac{r_{\max }}{r_{0}}\right)\right] H_{\mu \nu \lambda}^{0} \psi
$$

where we use the relation between the fundamental Planck scale $M$ and the 4-D Planck scale $M_{P l}$ as,

$$
M_{P l}=\frac{M^{3 / 2}}{\sqrt{2 R}} r_{\max }\left(1-\frac{r_{0}^{2}}{r_{\max }^{2}}\right)^{1 / 2}
$$

Eq.(18) is obtained by integrating the five dimensional gravitational action with respect to the internal component $r$ and comparing the derived effective 4-dimensional action with the standard 4-dimensional gravitational action. We have also used eq.(6) in order to obtain the final expression (i.e eq.(17)) for the coupling of the massless mode of the KR field with the SM fermion fields on the IR brane. It is now clear from eq.(17), that the coupling of the massless KR field with the SM fermions on the IR brane is heavily suppressed by an exponential factor which depends on the background fluxes.

\section{A. Estimation of the background fluxes}

Turning our attention to the experimental evaluation of four dimensional torsion components we see that in recent past the authors of [18 20] have considered a possible violation of CPT and local Lorentz invariance due to the presence of nonzero spacetime torsion in four dimensions. They have shown that torsion components can be constrained to have an upper bound of the order of $10^{-31} \mathrm{GeV}$. In this work, these experimental results 18 20] have been used to put bounds on various parameters of the model.
Equating the upper bound $10^{-31} \mathrm{GeV}$ on the 4dimensional torsion component as evaluated in [18 20] with the effective torsion component in the background of the KS geometry (see sectionII), we obtain the lower bound on the ratio of the background fluxes and string coupling constant to be $\frac{K}{g_{S} M} \sim 8.8$.

With this value for $\frac{K}{g_{S} M}$, eqn(6) yields a warp factor $\sim 10^{-10}$. In order to address the gauge hierarchy problem in this background geometry, the value of $\frac{K}{g_{S} M} \sim 17.5$ such that the warp factor $\sim 10^{-16}$. For this choice of the parameter $\frac{K}{g_{S} M}$, coupling becomes much more weaker which in turn reduces the possibility of having signatures of spacetime torsion in future experiments even more.

In summary this result depicts that in the background of the warped throat-like compactification the massless mode of the second rank antisymmetric KR field ans it's couplings are heavily suppressed on the IR region of the spacetime.

\section{ANALYSIS OF MASSLESS HIGHER RANK ANTISYMMETRIC TENSOR FIELDS IN THE KLEBANOV-STRASSLER GEOMETRY}

Here in the background of the KS throat geometry, we investigate the behaviour of massless higher rank antisymmetric tensor excitations of closed strings on the low energy /IR brane.

We consider a rank-3 antisymmetric tensor field, $X_{M N A}$, with the corresponding field strength tensor $Y_{M N A B}$. The five dimensional action of $X_{M N A}$ can be written as,

$$
S=\int d^{5} x \sqrt{-G} Y_{M N A B} Y^{M N A B}
$$

$\mathrm{G}$ is the determinant of the 5 dimensional $A d S_{5}$ metric. In general for a rank $n$ antisymmetric gauge field $X_{a_{1} a_{2} \ldots a_{n}}$, one should be able to write down a rank$(n+1)$ antisymmetric field strength tensor as,

$$
Y_{a_{1} a_{2} \ldots a_{n+1}}=\partial_{\left[a_{n+1}\right.} X_{\left.a_{1} a_{2} \ldots a_{n}\right]}
$$

Using the explicit form of the five dimensional metric (eq.(2)), the gauge fixing condition for the tensor field $X\left(\right.$ i.e $\left.X_{\mu \nu r}=0\right)$ and the KK decomposition of the field $\mathrm{X}$, we obtain the effective 4-dimensional action for the tensor field $X$,

$$
\begin{array}{r}
S_{x}=\int d^{4} x \sum_{n}\left[\eta^{\mu \lambda} \eta^{\nu \rho} \eta^{\alpha \gamma} \eta^{\beta \delta} Y_{\mu \nu \alpha \beta}^{n} Y_{\lambda \rho \gamma \delta}^{n}\right. \\
\left.+4 m_{n}^{2} \eta^{\mu \lambda} \eta^{\nu \rho} \eta^{\alpha \delta} X_{\mu \nu \alpha}^{n} X_{\lambda \rho \gamma}^{n}\right]
\end{array}
$$

where $m_{n}^{2}$ is defined through the relation,

$$
-\partial_{r}^{2} \chi^{n}(r)=m_{n}^{2} h(r) \chi^{n}(r)
$$

and $\chi^{n}$ satisfies the orthonormality condition,

$$
\frac{1}{r_{c}} \int_{r_{0}}^{r_{\max }} h^{5 / 4}(r) \chi^{m}(r) \chi^{n}(r) d r=\delta_{m n}
$$


Proceeding similarly as in case of the KR field in the preceding section, we obtain the wavefunction for the massless mode of the tensor field $X$ on the IR brane located at $r=r_{0}$,

$$
\chi^{0}=2 e^{-5 \pi K / 3 g_{s} M}\left(\frac{r_{\max }^{2}}{r_{0}^{2}}\right)\left[1+\frac{r_{\max }^{2}}{r_{0}^{2}}\right]^{-1 / 2}\left(1+\frac{r_{\max }}{r_{0}}\right)^{-1 / 2}
$$

We evaluate the interaction Lagrangian of the massless mode of the third rank antisymmetric tensor gauge fields with the SM fermion fields on the low energy brane, located at $r=r_{0}$ as,

$$
\begin{gathered}
\mathscr{L}_{\psi \bar{\psi} Y^{0}}=i \bar{\psi} \gamma^{\alpha} \Sigma^{\mu \nu \lambda} \sqrt{2}\left(\frac{e^{-2 \pi K / g_{s} M}}{M_{P l}}\right)\left(\frac{r_{\max }^{2}}{r_{0}^{2}}\right) \\
{\left[1+\frac{r_{\max }^{2}}{r_{0}^{2}}\right]^{-1 / 2} Y_{\mu \nu \alpha \beta}^{0}\left(x^{\mu}\right) \psi}
\end{gathered}
$$

Thus, in the background of the KS throat geometry, the massless mode of the higher rank tensor gauge field and it's coupling with the SM fermions on the IR brane is even more suppressed than the massless mode of the second rank antisymmetric KR field.

This analysis can easily be extended to even higher rank antisymmetric gauge fields which exhibits even higher suppression factor for all of them.

\section{CONCLUSION}

String theory predicts the presence of massless modes of various higher rank antisymmetric tensor fields. An inevitable question that arises is, why all these massless modes of these tensor fields are invisible in our Universe? Here we try to address this question in the background of a warped throat-like KS string compactification. We consider various higher rank tensor fields in the background of the KS geometry and analyse the massless modes of these tensor fields projected on our brane.

Our result reveals that the massless modes of all these higher rank tensor gauge fields are heavily suppressed on the IR region of the spacetime which can be depicted as the SM brane/our Universe. In case of the KR field which also can act as the spacetime torsion, is exponentially suppressed on the SM brane, where the amount of suppression is parametrised by the background fluxes of the spacetime. We also show that the higher rank antisymmetric tensor gauge fields are even more suppressed than the rank two antisymmetric KR field. In the context of the rank two antisymmetric tensor field which is also interpreted as the spacetime torsion, we estimate the lower bound on the ratio of background fluxes and string coupling constant by incorporating the experimental upper bound on the 4-dimensional torsion component.

Looking back to earlier works, where all the higher rank tensor gauge fields have been analysed in the context of the warped RS scenario in five dimensions, it can be stated in general that a warped nature of spacetime, in a string-inspired scenario can also explain the absence of any signatures of higher rank antisymmetric tensor fields in our Universe for appropriate choices of background fluxes.
[1] P.Majumdar,S.SenGupta, Classical Quantum Gravity 16, L89 (1999)

[2] B.Mukhopadhyaya,S.Sen,S.SenGupta, Phys.Rev.Lett.89:121101,2002

[3] L. Randall and R. Sundrum, Phys. Rev. Lett. 83, 3370 (1999); ibid 83, 4690 (1999).

[4] B.Mukhopadhyaya,S.Sen,S.SenGupta, Phys.Rev. D76 (2007) 121501

[5] I.R. Klebanov and M.J. Strassler, J. High Energy Phys. 08, 052 (2000), hep-th/0007191

[6] H. Verlinde, Nucl. Phys. B580, 264 (2000), hep-th/9906182

[7] Shamit Kachru1, Renata Kallosh, Andrei Linde, Juan Maldacena, Liam McAllister and Sandip P Trivedi, JCAP 0310 (2003) 013.

[8] Alex Buchel, Radu Roiban, Physics Letters B 590 (2004) 284294

[9] Daniel Baumann and Liam McAllister, Phys. Rev. D75, 123508 (2007)
[10] Rachel Bean, Sarah E Shandera, S-H Henry Tye and Jiajun Xu, JCAP 0705:004,2007, arXiv:hep-th/0702107 1

[11] Gary Shiu and Bret Underwood, Phys. Rev. Lett. 98, 051301 (2007)

[12] Lev Kofman and Shinji Mukohyama, Phys. Rev. D 77, 043519 (2008)

[13] Daniel Baumann, Anatoly Dymarsky, Shamit Kachru, Igor R. Klebanovb and Liam McAllister, JHEP 0903:093,2009, arXiv:0808.2811 v3 [hep-th]

[14] Daniel Baumann, Liam McAllister, "Inflation and String Theory", arXiv:1404.2601 v1 [hep-th].

[15] Michael R. Douglas, S. Kachru, "Flux compactification", REVIEWS OF MODERN PHYSICS, 79, 2007.

[16] R. Kallosh, A. Linde, and T. Rube, Phys.Rev. D83 (2011) 043507

[17] Steven B. Giddings, Shamit Kachru and Joseph Polchinski1, Phys. Rev. D 66, 106006 (2002).

[18] F. Cane' et al., Phys. Rev. Lett. 93, 230801 (2004).

[19] B. R. Heckel et al., Phys. Rev. Lett. 97, 021603 (2006).

[20] V. Alan Kostelecky,Neil Russell and Jay D. Tasson1, Phys. Rev. Lett. 100, 111102 (2008). 